\title{
Análisis de la competencia digital docente del profesorado de colegios rurales agrupados de la provincia de Albacete
}

\section{Analysis of the digital teaching competence of teachers of rural schools in the province of Albacete}

\author{
María del Pilar Ruiz Ruiz \\ CRA de Castilla-La Mancha \\ mariapi17rr@hotmail.com
}

Recibido: $11 / 09 / 2019$

Aceptado: $18 / 02 / 2020$

Publicado: 01/06/2020

\section{RESUMEN}

La transmisión de la cultura digital es uno de los retos más destacados del actual sistema educativo. La Unión Europea se plantea a través de la Estrategia Europa 2020 mejorar la accesibilidad, el uso y la calidad de las Tecnologías de la Información y de la Comunicación (TIC) en las zonas rurales. Para que ello sea posible, se precisan de recursos materiales y profesionales digitalmente competentes. En este estudio se pretende conocer la realidad que viven las escuelas rurales, de la provincia de Albacete, en lo que respecta a la calidad de la TIC, los recursos disponibles y las competencias digitales de sus docentes. Para ello, se ha empleado una metodología descriptiva y se ha utilizado el cuestionario como instrumento de recogida de información. Los resultados demuestran que las intenciones políticas no concuerdan con la realidad encontrada en nuestros datos. Las infraestructuras tecnológicas carecen de la calidad suficiente que posibiliten un acceso a internet de forma segura y eficaz. Por otro lado, los docentes que desempeñan su labor en estos centros hacen un uso limitado de las tecnologías en estas aulas, caracterizadas por ser multinivel, debido a la falta de formación en competencias digitales.

\section{PALABRAS CLAVE}

TIC; escuela; competencias docentes.

\section{ABSTRACT}

The transmission of digital culture is one of the most important challenges of the current education system. The European Union proposes, through the Europe 2020 Strategy, to improve the accessibility, use and quality of Information and Communication Technologies (ICT) in rural areas. For this to be possible, digitally competent material and professional resources are required. This study aims to know the reality of rural schools in the province of Albacete, in terms of the quality of ICT, the available resources and the digital skills of their teachers. For this, a descriptive methodology has been used and the questionnaire has been used as an information collection instrument. The results show that the political intentions do not match the reality found in our data. Technological infrastructures lack sufficient quality to enable internet access in a safe and efficient way. On the other hand, teachers who carry out their work in these centers make limited use of the technologies in these classrooms, characterized by being multilevel, due to the lack of training in digital skills.

\section{KEYWORDS}

ICT; school; teacher qualifications 


\section{CITA RECOMENDADA}

Ruiz, M.P. (2020). Análisis de la competencia digital docente del profesorado de colegios rurales agrupados de la provincia de Albacete. Revista Interuniversitaria de Investigación en Tecnología Educativa, 8, 1-13. http://dx.doi.org/10.6018/riite.395721

\section{Principales aportaciones del artículo y futuras líneas de investigación:}

- Este articulo aporta una visión general sobre la situación de las escuelas rurales en cuanto a dotación tecnológica y acceso a las TIC. Por otro lado, describe y analiza cómo es la competencia digital de los docentes de los Colegios Rurales Agrupado, determinando la frecuencia con que ponen en práctica las competencias asociadas a cada área competencial, del marco INTEF.

- Como futuras líneas de investigación se pueden desarrollar estudios, con una muestra más amplia, que traten de comparar las escuelas rurales y urbanas, en cuanto a dotación tecnológica y competencia digital docente.

\section{INTRODUCCIÓN}

Las Tecnologías de la Información y de la Comunicación han llegado a nuestra sociedad de forma lenta y progresiva a lo largo del último tercio del siglo XX. La llegada de éstas al medio rural ha supuesto un soplo de aire fresco en la medida que se ha creado la oportunidad de romper con el aislamiento geográfico. Concretamente, la incorporación de las tecnologías en el ámbito educativo rural ha contribuido a crear oportunidades de acceso al conocimiento y la comunicación, dotando al sistema escolar de calidad (Salinas \& Sánchez, 2009).

Por lo tanto, una de las misiones fundamentales de los Colegios Rurales Agrupados (CRAs) es la de contribuir a la reducción de la brecha digital entre el mundo rural y urbano, mediante la transmisión de saberes tecnológicos y la adquisición de habilidades digitales por parte de los alumnos y sus familias (Elboj et al., 2013).

Para que este acceso a la nueva sociedad del conocimiento sea una realidad, se precisan de los siguientes factores: recursos físicos: recursos tecnológicos y conexión en las escuelas; recursos digitales: diversidad de contenidos; recursos sociales: familias y agentes sociales que contribuyen y apoyan el uso eficaz de las TIC y recursos humanos: competencias y conocimientos para el manejo de dispositivos e Internet (Warschauer, 2008).

Las posibilidades que ofrecen las TIC no sólo se manifiestan en el plano tecnológico sino también pedagógico, y es que el uso de las tecnológicas contribuye a la mejora de calidad de la educación a través de prácticas colaborativas e inclusivas, como, por ejemplo, "la Comunicación Virtual Intercentros; el Acceso a Materiales Remotos de Aprendizaje (promoviéndose un nuevo tipo de aprendizaje interactivo, más rápido y profundo, de mayor calidad); el Establecimiento de Comunidades Profesionales On-Line y la Desprivatización de la Práctica Docente"(MartínMoreno, 2002, p. 64).

Sin embargo, la incorporación de las TIC a la escuela rural se ve dificultada por la falta de habilidades docentes en TIC (Carnoy, 2004). Por lo tanto, las aulas precisan de profesionales alfabetizados digitalmente, es decir docentes competentes digitalmente, que cuenten con las capacidades, actitudes, destrezas, creencias y valores que les posibiliten para utilizar de forma adecuada las tecnologías, con el fin de acceder y utilizar la información para construir conocimiento (Gutiérrez, 2011).

En esta línea, es necesario hacer mención del Instituto Nacional de Tecnologías Educativas y Formación del Profesorado (INTEF), organismo que tiene entre sus misiones fundamentales la de formar a docentes en competencias digitales, para asegurar una docencia de alta calidad de acuerdo con los objetivos establecidos en el "Marco estratégico europeo de Educación y Formación 2020". 
De este modo, se ha tomado como referente, para analizar la competencia digital docente la clasificación del portfolio del INTEF (2017), que se divide en cinco áreas: Información y Alfabetización Informacional, Comunicación y Colaboración, Creación de Contenido Digital, Seguridad y Resolución de problemas.

\subsection{Proceso de digitalización de las aulas de Castilla-La Mancha}

Siguiendo el Portal de Tecnología en Educación, de la Junta de Comunidades de CastillaLa Mancha, el proceso de integración del TIC en las aulas rurales, comenzó en el curso 19971998, a través del proyecto Aldea Digital, el cual acercó internet a los colegios rurales. El Misterio de Educación señaló como beneficioso de este proyecto el acercamiento de las TICS al mundo rural, permitiendo hacer frente a la incomunicación que durante años había afectado a estas zonas.

Años más tarde, a partir del curso 2004-2005, se empezaron a instalar en las aulas de todos Ios IES las Aulas Althia, dotadas con medios para la información y Comunicación, que permitieron desarrollar entornos de trabajo colaborativo entre alumnos y profesores a través del Plan de Conectividad Integral.

Posteriormente, en el período 2009-2012, se pone en marcha el programa de integración de la Escuela 2.0, una iniciativa gubernamental de innovación educativa, en colaboración con las Comunidades Autónomas, que trató de poner en funcionamiento el Aula Digital del siglo XXI dotadas de infraestructuras tecnológicas y de conectividad. Su finalidad fue digitalizar progresivamente todas las aulas de los centros de primaria y secundaria, haciendo uso de las TIC como una herramienta más del proceso de aprendizaje del alumnado. Entre sus objetivos se encontraba asegurar la formación del profesorado, técnica y metodológicamente, la integración de los recursos en el aula, como la Pizarra Digital Interactiva (PDI), un videoproyector para su uso con la anterior pizarra, un ordenador netbook para su uso con cada alumno del aula, un mueble o maletín de custodia (en función del número de alumnos) para albergar los netbooks del alumnado, un ordenador portátil para el profesorado y conexión a Internet WI-FI.

Para acceder a información relativa a los centros educativos existen los portales Web de Centros Educativos 3.0. A través de ella cualquier usuario que acceda, podrá obtener información sobre su oferta académica, entre otros aspectos.

Recientemente el Gobierno Regional ha distribuido una gran cantidad de ordenadores portátiles para el profesorado de los centros educativos públicos castellanomanchegos. Se trata de una medida que se equipara con otras comunidades. Concretamente, dentro del Plan Escuela TIC 2.0, durante el curso 2009/2011, se distribuyeron miles de ordenadores portátiles destinados a docentes de las aulas andaluzas.

Para desarrollar los procesos de gestión administrativa y académica de los centros educativos de la región castellanomanchega, existe el programa Delphos.

Por otro lado, la plataforma educativa Papás 2.0, permite efectuar los procesos de gestión administrativa de los ciudadanos, así como llevar a cabo una comunicación fluida entre los centros educativos y las familias, ofreciendo información a todos los agentes de la comunidad educativa. Dispone de una secretaría virtual, un aula virtual y un área de comunicación para/con las familias.

Actualmente ha surgido un proyecto innovador que tiene como objetivo digitalizar las aulas de los centros educativos públicos de Castilla-La Mancha, llamado Proyecto Carmenta. Se trata de un sistema de ayudas que trata de promover el uso de tabletas por parte del alumnado y del profesorado. Esta medida va encaminada a sustituir el libro de texto en préstamo para incorporar las tabletas igualmente en préstamo, las cuales llevarán incorporadas las licencias de los libros digitales que le correspondan en función del grado de ayuda concedida.

Por último, la herramienta Territorio Cuadernia, permite la creación y difusión de materiales educativos digitales por parte de toda la comunidad educativa. Entre sus aplicaciones destaca el Editor Cuadernia, la Biblioteca ODEs, Cuadenia Social y estándares de Cuadernia. 
A continuación, en la siguiente tabla, se muestra a modo de síntesis los proyectos digitales que hemos mencionado, ordenados de manera cronológica según el año de incorporación y destacando las características más significativas de cada uno de ellos.

Tabla 1. Histórico de digitalización de centros escolares en Castilla-La Mancha

\begin{tabular}{|c|c|c|}
\hline Año & Nombre & Descripción \\
\hline 1997 & Proyecto Aldea Digital & Incorporación de las TIC a la escuela rural. \\
\hline 2004 & Aulas Athia & $\begin{array}{l}\text { Infraestructura tecnológica. Plan de conectividad } \\
\text { integral. Entornos de trabajo colaborativos. }\end{array}$ \\
\hline 2004 & Programa Delphos & $\begin{array}{l}\text { Gestión administrativa y académica de centros } \\
\text { educativos. }\end{array}$ \\
\hline 2009 & Escuela 2.0 & $\begin{array}{l}\text { Aula Digital. Dotación de infraestructura tecnológica y } \\
\text { conectividad. }\end{array}$ \\
\hline 2009 & $\begin{array}{l}\text { Web centros educativos } \\
3.0\end{array}$ & Acceso a información del centro. \\
\hline 2010 & Papás 2.0 & $\begin{array}{l}\text { Gestión administrativa. Comunicación familia y centro } \\
\text { educativo. Secretaría y aulas virtuales. }\end{array}$ \\
\hline 2012 & Territorio Cuadernia & Creación y difusión de materiales educativos digitales \\
\hline 2018 & Proyecto Carmenta & $\begin{array}{l}\text { Préstamo de tabletas digitales y licencias de libros } \\
\text { digitales. }\end{array}$ \\
\hline
\end{tabular}

\section{MÉTODO}

\subsection{Problema de investigación}

Después de la revisión bibliográfica se han planteado las siguientes preguntas que darán respuesta al problema de investigación:

- ¿Están las Escuelas Rurales de Albacete, capacitadas en recursos para el acceso al mundo tecnológico?

- ¿Son los docentes de las escuelas rurales, de Albacete, competentes digitalmente?

\subsection{Objetivos}

Para dar respuesta a estas preguntas se han establecido los siguientes objetivos:

Objetivo general:

- Conocer la realidad en cuanto a dotación tecnológica y competencia digital docente en el ámbito de la escuela rural, en la provincia de Albacete.

Objetivos específicos:

- Describir y analizar las infraestructuras tecnológicas con las que cuentan los Colegios Rurales Agrupados de la provincia de Albacete y los usos que el profesorado hace de ellas.

- Describir y analizar cómo es la competencia digital docente de los maestros de los Colegios Rurales Agrupados de la provincia de Albacete.

\subsection{Diseño de la investigación}

Los datos recabados en este estudio nos han permitido responder a las preguntas de investigación. Por un lado, describir y analizar las infraestructuras tecnológicas con las que cuentan los Colegios Rurales Agrupados de la provincia de Albacete y los usos que el profesorado hace de ellas; por otro lado, describir y analizar cómo es la competencia digital docente de los maestros de los Colegios Rurales Agrupados de la provincia de Albacete. Por lo tanto, este estudio se enmarca en una metodología no experimental y de corte descriptivo, ya 
que su principal objetivo es conocer una realidad, unas concepciones y unas características de una pequeña muestra. El enfoque metodológico es de naturaleza cuantitativa, empleando es cuestionario como técnica de recogida de información y posterior análisis de datos a través de porcentajes y frecuencias.

\subsection{Participantes}

Nuestro estudio está formado por una muestra perteneciente a cinco CRA, que son los siguientes: CRA Río Mundo (Agramón); CRA San José (Molinicos) ; CRA Río Taibilla (Nerpio); CRA Yeste (Yeste); CRA Calar del Mundo (Riópar)

El tipo de muestreo ha sido por conveniencia, por lo tanto, es no probabilístico. El número total de participantes ha sido de 44 maestros.

Con respecto a las características de los participantes, el $70 \%$ de los encuestados pertenecen al sexo femenino y el $30 \%$ al sexo masculino. El $30 \%$ tienen edades comprendidas entre los 31 y 40 años; el $27 \%$ entre los 26 y los 30 años; el 18\% se sitúan entre los 20 y los 25 años; el 14\% entre los 41 y los 50 años; y el $9 \%$ tienen más de 51 años. Los participantes cuentan con titulaciones propias de los maestros de Colegios de Infantil y Primaria: Magisterio en Educación Primaria, Magisterio en Educación Infantil, Magisterio en Lenguas Extranjeras, Magisterio en Educación Física, Magisterio en Educación Musical, Magisterio en Pedagogía Terapéutica y Magisterio en Audición en Lenguaje. En cuanto al tipo de contrato la mayoría de ellos, un $65,9 \%$ son interinos; un $31,8 \%$ son funcionarios de carrera y un $2,3 \%$ son funcionarios en prácticas. En cuanto a los años de experiencia docente el $47,7 \%$ cuentan con menos de tres años de experiencia; el $25 \%$ tienen entre tres y ocho años de experiencia; $13,6 \%$ tienen entre ocho y 20 años de experiencia; y el 13,6\% tienen más de veinte años de experiencia docente.

\subsection{Instrumento de recogida de datos}

Como instrumento de recogida de información se ha elaborado un cuestionario para su difusión e implementación por vía digital (ver en apartado 5).

El cuestionario se ha dividido en cuatro bloques integrado por 37 ítems, organizados de la siguiente manera:

- Bloque I. Demográfica. En este apartado sean recogido aspectos relativos a la edad, sexo, titulación académica, años de experiencia docente.

- Bloque II. Recursos disponibles. Compuesto por preguntas relativas a el tipo de conexión internet, el tipo de recursos tecnológicos que utilizan en sus clases, y su frecuencia de uso.

- Bloque III. Formación docente. Las preguntas hacen referencia a la formación en nuevas tecnologías, si ha participado en los últimos meses en algún proyecto o curso relacionado con la mejora de las competencias digitales del profesorado, además de si considera importante la formación permanente en nuevas tecnologías.

- Bloque IV. Competencias digitales. El resto del cuestionario nos ha proporcionado información referente a competencias digitales docentes, en base al marco DIGCOMP 2.0.

\subsection{Análisis de datos}

De acuerdo con la naturaleza de los datos, para el análisis de resultados se ha empleado la estadística descriptiva, en función de los objetivos, el análisis de datos se muestra a través de frecuencias y porcentajes, lo cuales, sintetizan de manera clara y concisa las respuestas de los participantes. 


\section{RESULTADOS}

\subsection{Conexión a internet}

Como se puede observar en la Figura 1 , el $34,1 \%$ de los docentes se conectan a internet mediante redes inalámbricas; el $20,5 \%$ a través de satélite; el 18,2 \% por medio de línea telefónica ; el 13,6 \% con fibra óptica; Además, hay un 9,1\% de encuestados que no conocen el tipo de conexión a Internet.

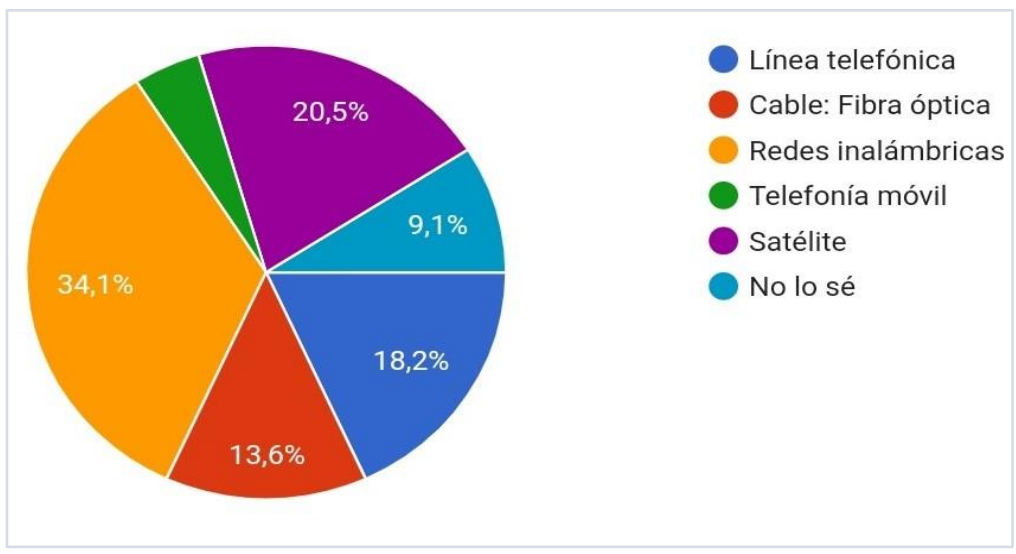

Figura 1. Tipo de conexión a internet

\subsubsection{Disponibilidad de banda ancha}

En cuanto a la disponibilidad de banda ancha, si observamos la Figura 2, la mayoría de los encuestados, el $43,2 \%$ destacan que el centro no dispone de esta funcionalidad; sin embargo, el $34,1 \%$ afirma que su centro sí que dispone de ella. Hay un porcentaje de $22,7 \%$ que desconoce si su centro dispone de banda ancha para la conexión a internet.

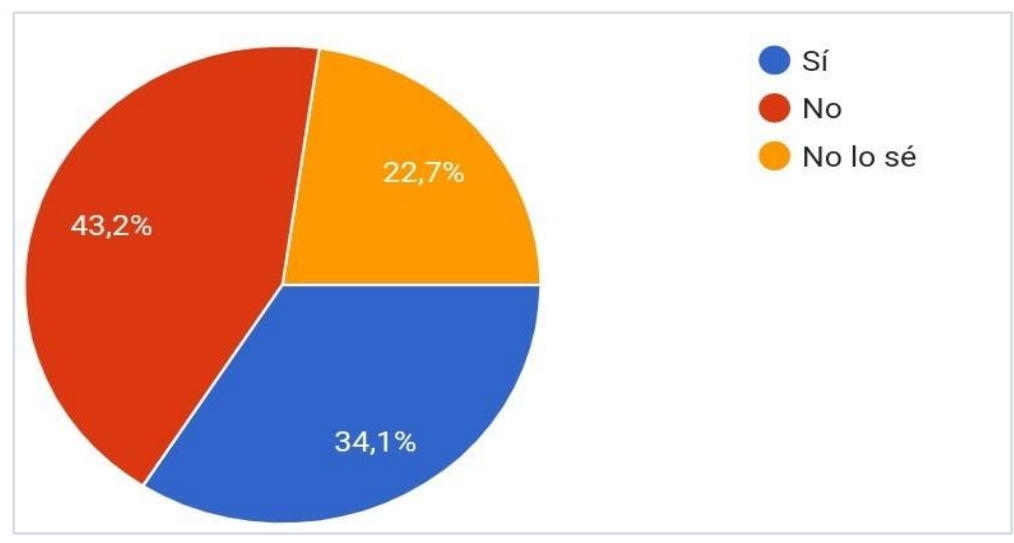

Figura 2. Disponibilidad banda ancha

\subsection{Recursos tecnológicos}

\subsubsection{Recursos tecnológicos disponibles en el aula}

Los resultados de la figura 3 y 4 , demuestran que el ordenador portátil es el recurso tecnológico que mayoritariamente utilizan los profesores en sus aulas, seguidos de los proyectores, las tablets, la pizarra digital interactiva, el ordenador fijo y finalmente el smartphone. 


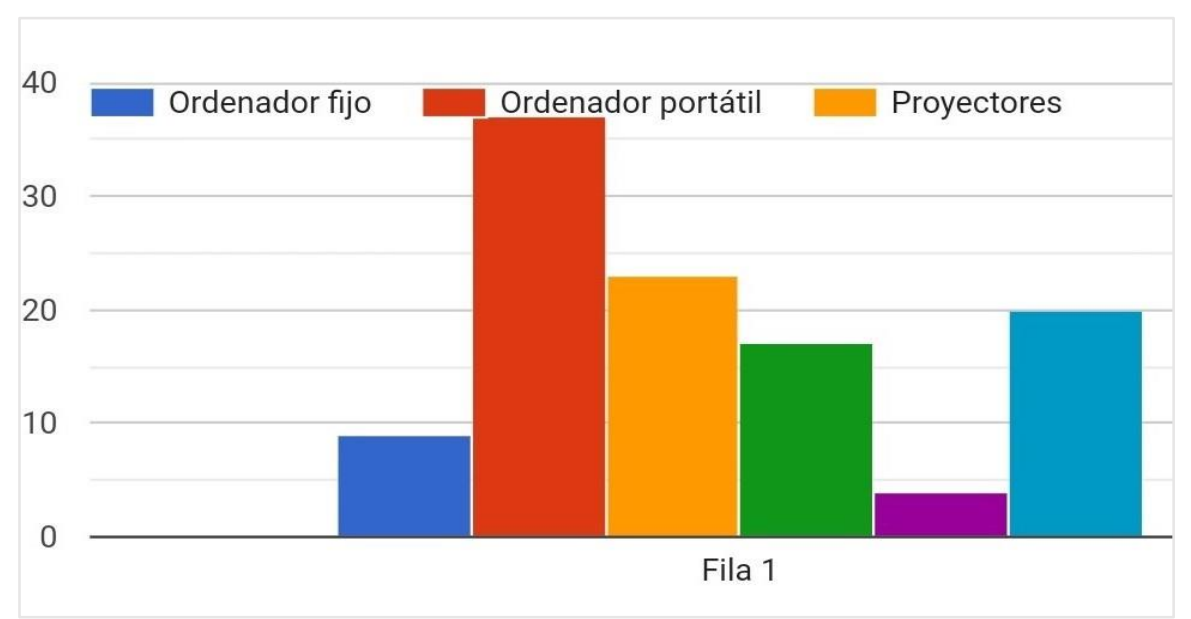

Figura 3. Recursos tecnológicos en aula 1/2

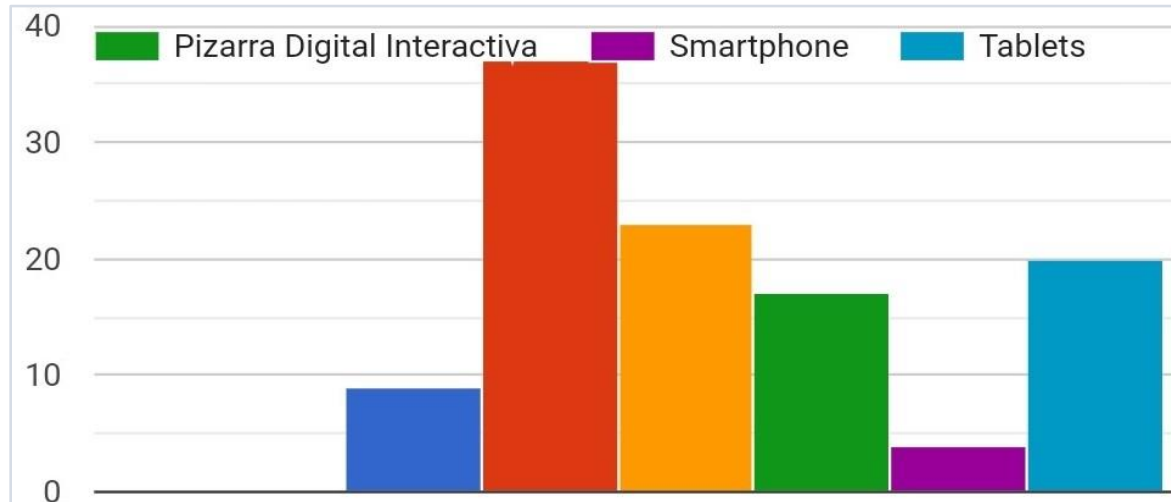

Fila 1

Figura 4. Recursos tecnológicos en el aula 2/2

\subsubsection{Frecuencia de uso}

La figura 6, en el análisis de resultados demuestra que la mayoría de los profesores, un $45,5 \%$ utiliza estos recursos en sus aulas de forma ocasional; el $25 \%$ casi nunca hace uso de ellos; el 13,6 \% los utiliza de manera ocasional; y el 15,9\% siempre hace uso de ellos en el aula.

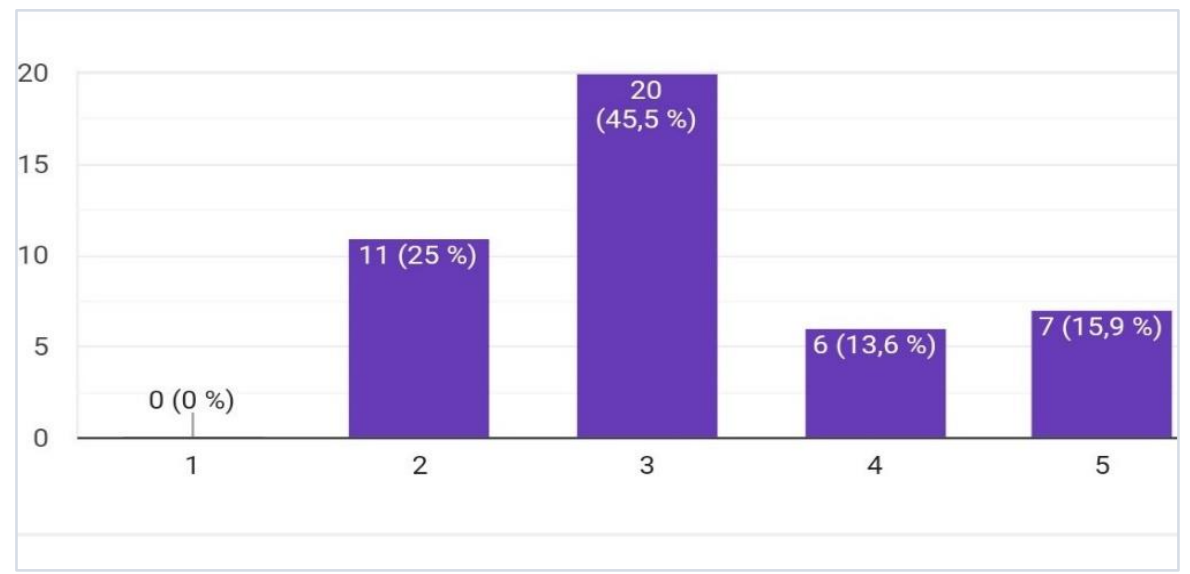

Figura 5.Frecuencia de uso de recursos tecnológicos 


\subsection{Formación en nuevas tecnologías}

\subsubsection{Formación en nuevas tecnologías durante los estudios universitarios}

Siguiendo la figura 7 , el $67,4 \%$ de los docentes se ha formado en nuevas tecnologías durante sus estudios universitarios; Sin embargo, el 32,6 \% dice no haber cursado estudios relacionados con las enseñanzas tecnológicas.

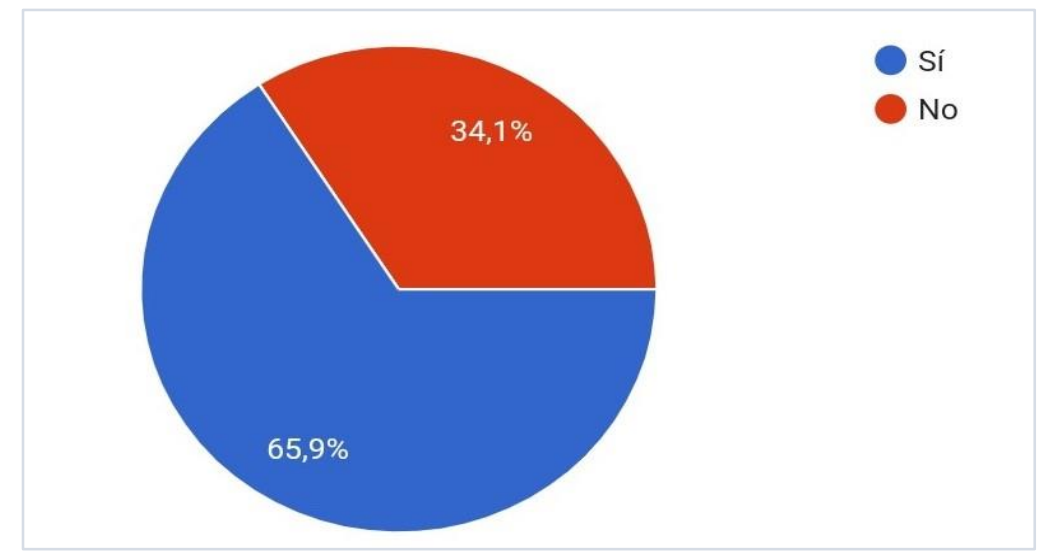

Figura 6. Formación en TIC durante estudios universitarios

\subsubsection{Actualización docente en nuevas tecnologías}

Con lo que respecta a la participación de los encuestados en cursos, jornadas o seminarios relacionados con las tecnologías, que impliquen una mejora de las competencias TIC, la figura 7 , muestra que el $45,5 \%$ dice haber participado en los últimos meses en alguna acción formativa en TIC, frente al 54,5\% que destaca no haber recibido enseñanza alguna.

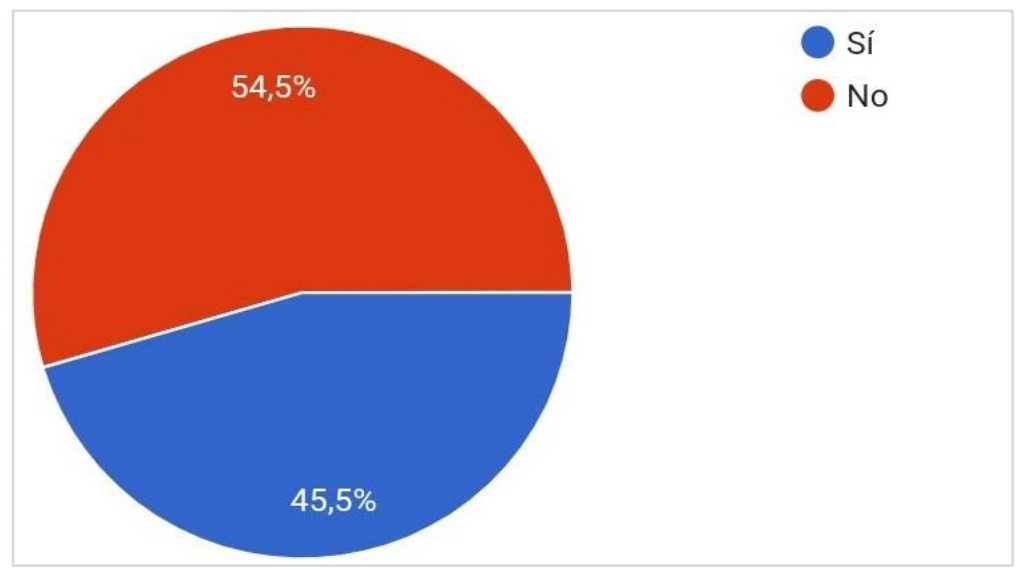

Figura 7. Actualización docente en TIC

\subsection{Competencias digitales docentes}

\subsubsection{Informatización y alfabetización informacional}

Navegación, búsqueda y filtrado de información, datos y contenido digital.

En lo que respecta a al desarrollo de habilidades de búsqueda de información en red, la Figura 8 muestra que un $25,6 \%$ de los encuestrados siempre desarrolla y hace uso de habilidades para buscar información digital; el mayor porcentaje de respuestas se sitúa en la búsqueda casi constante y constante de información con un 27,9\% de los encuestados, con 
igual porcentaje en ambas respuestas. Un 16,3\% destacan una búsqueda en red muy ocasional y tan sólo un 2,3 \% afirma que nunca utiliza este sistema digital para la navegación.

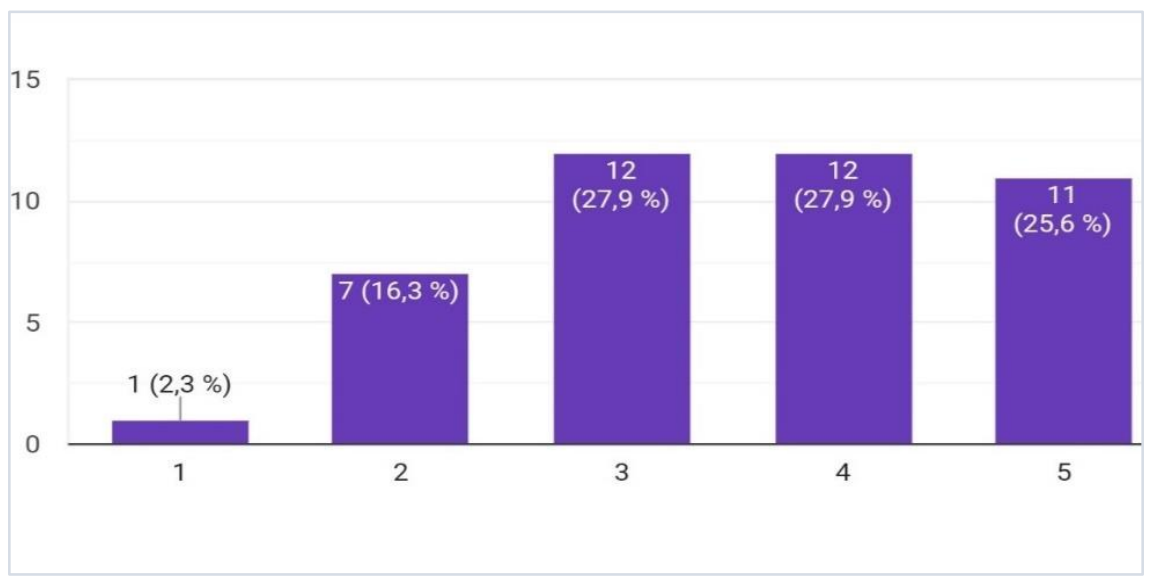

Figura 8. Búsqueda de información en red

\subsubsection{Comunicación y colaboración}

Interacción mediante tecnologías digitales.

En esta cuestión se les pregunta a los docentes con qué frecuencia utilizan entornos de trabajo digitales para la interacción con el alumnado y sus familias, a través de correo electrónico, teléfono móvil, chat, etc. Los resultados, de la Figura 9, demuestran que el $29,5 \%$ de los encuestados casi siempre utiliza herramientas digitales para la comunicación; el 22,7 \% en ocasiones; el 18,2 \% casi nunca; el 15,9 \% nunca hace uso de ellas para interaccionar con otros; y el 13,6 \% siempre las utiliza para compartir información.

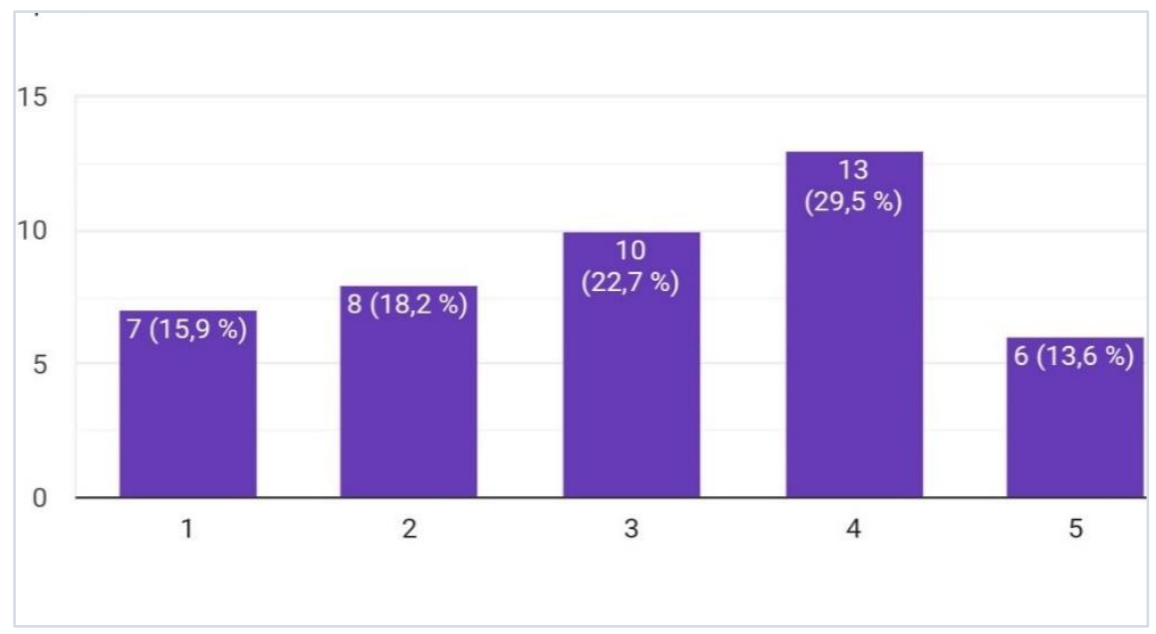

Figura 9. Comunicación a través de tecnologías digitales

\subsubsection{Creación de contenido digital}

\section{Desarrollo de contenidos digitales}

En lo que respecta a la creación de contenidos digitales en diferentes formatos multimedia, por ejemplo, texto, audio, imagen, etc., para su uso en el aula, los resultados de la Figura 10, destacan que un $32,6 \%$ casi nunca elabora representaciones de conocimiento usando medios digitales; un $25,6 \%$ casi siempre; un $18,6 \%$ en ocasiones; un $14 \%$ nunca y un $9,3 \%$ siempre crea contenidos digitales en formatos multimedia. 


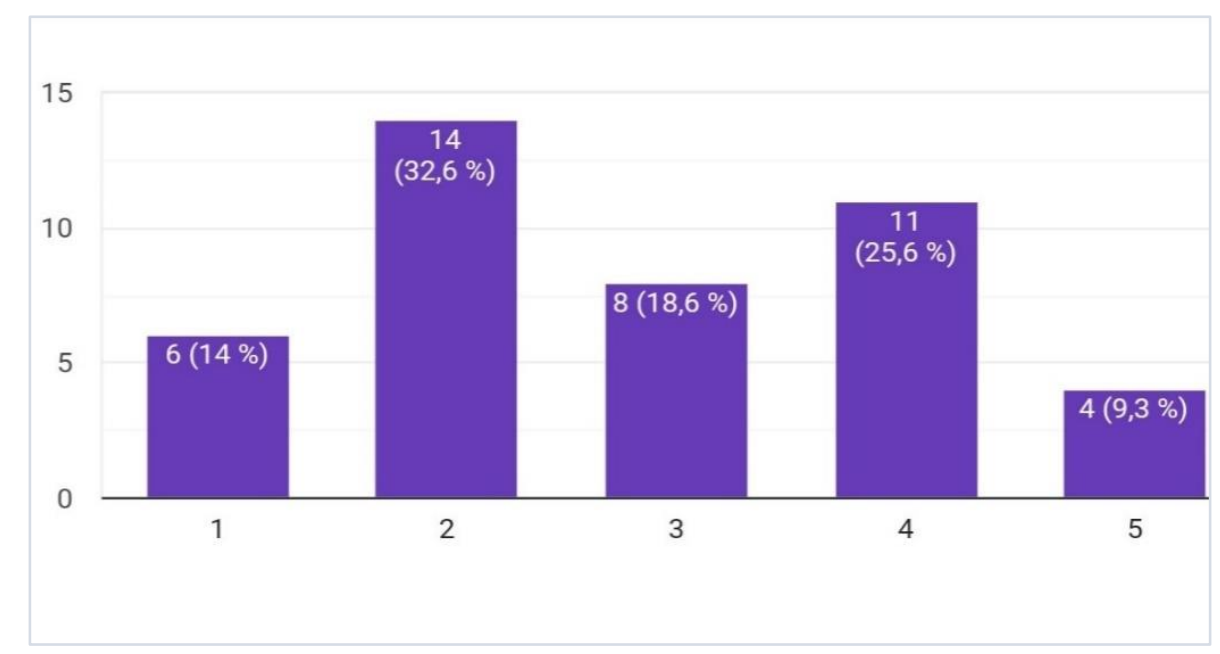

Figura 10. Creación de contenidos digitales

\subsubsection{Seguridad}

Protección de datos personales e identidad digital

Con respecto a la cuestión de si mantienen una actitud activa destinada a proteger la privacidad y evitar riesgos en red asociados a fraudes o ciberacoso, la Figura 11 muestra que el mayor porcentaje de respuesta si sitúa en el concepto de casi nunca; seguido del concepto de siempre, con un $25 \%$; a continuación ocasionalmente, ha sido contestado por el $22,7 \%$ de los encuestados; casi siempre ha sido respondido por el $20,5 \%$ de los docentes; y un $4,5 \%$ afirma no tener nunca una actitud activa para la protección de la privacidad.

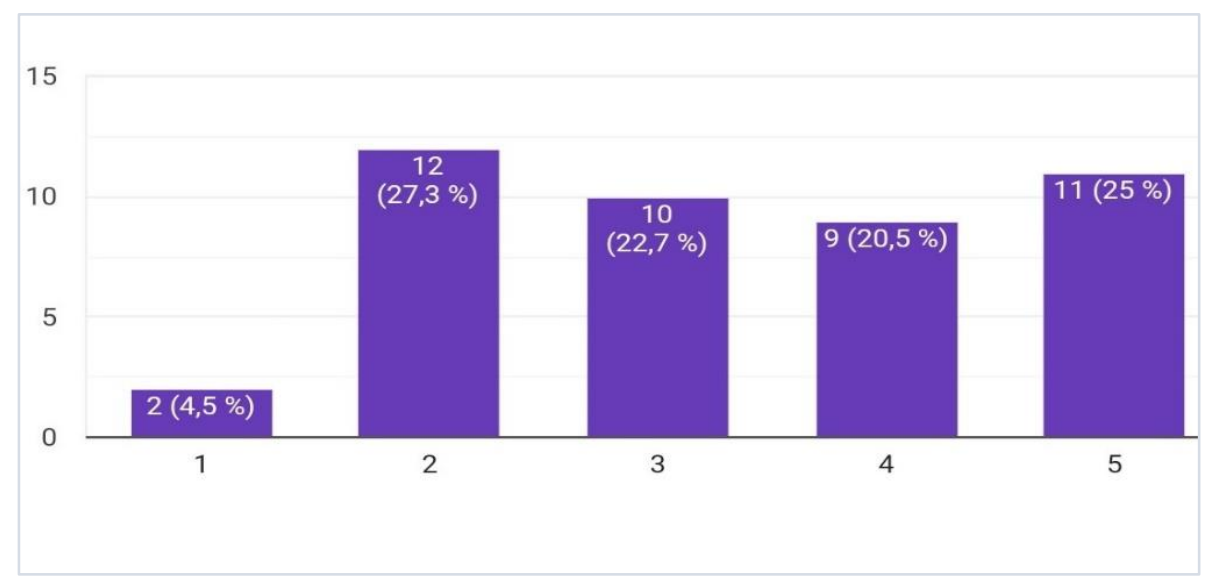

Figura 11. Protección de datos

\subsubsection{Resolución de problemas}

Innovación y uso de la tecnología digital de forma creativa

Para conocer si los alumnos son capaces de utilizar la tecnología digital de forma creativa, elaborando productos innovadores, de carácter significativo para los alumnos, los datos de la Figura 13, demuestran que el $36,4 \%$ casi constantemente desarrolla esta competencia; el $25 \%$ 
lo hace muy ocasionalmente; el $20,5 \%$ ocasionalmente; el $15,9 \%$ siempre; y el 2,3 \% nunca participa en acciones innovadoras de creación de conocimiento.

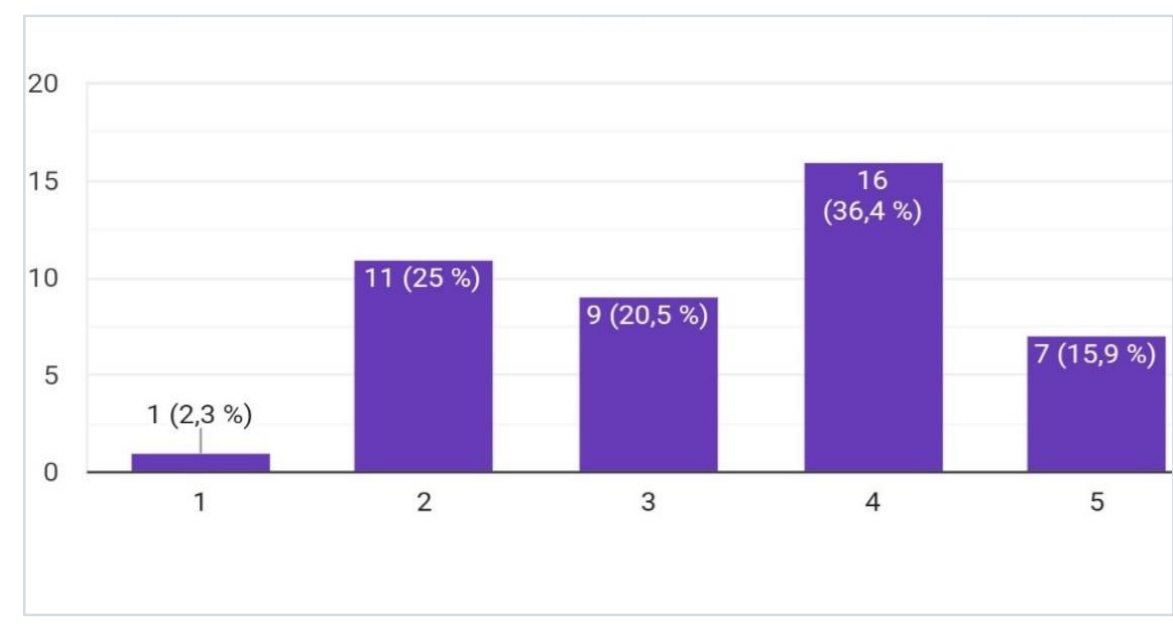

Figura 12. Innovación digital

\section{DISCUSIÓN Y CONCLUSIONES}

Las conclusiones de este estudio darán respuesta a los objetivos de investigación, por lo que se establece lo siguiente:

La calidad de la conexión internet es percibida como mala, debido a que la mayoría de los centros no disponen de banda ancha. En el marco teórico, hemos hecho referencia a que una de las misiones fundamentales de los Colegios Rurales Agrupados, es contribuir a la reducción de la brecha digital entre el mundo rural y urbano (Elboj et al., 2013). Por otro lado, la Unión Europea se plantea a través de la Estrategia Europa 2020 mejorar la accesibilidad, el uso y la calidad de las Tecnologías de la Información y de la Comunicación (TIC) en las zonas rurales. Los resultados demuestran una desigualdad en el acceso a la Sociedad de la Información entre el mundo rural y urbano y por consiguiente un incumplimiento de los principios establecidos a nivel europeo.

En cuanto al recurso tecnológico que más se utiliza en el aula destaca el ordenador portátil, seguido de los proyectores, tablets, pizarra digital interactiva, ordenador fijo y smartphone. La utilización de estos recursos viene impulsada por el proceso de Escuela 2.0 en el cual se pretende integrar todos estos medios digitales en el proceso de enseñanza. Sin embargo, la frecuencia de uso de estos recursos en el aula es limitada. Esto demuestra que no se utilizan de manera habitual en el aula, ello puede ser debido a causas como la baja calidad de la conectividad a la red, la falta de formación, de interés, de tiempo, o incluso volviendo a razones administrativas, la ausencia de regulaciones o incentivos que muevan a los docentes a integrarlas en sus prácticas habituales.

En cuanto al segundo objetivo de este estudio, se ha buscado describir y analizar cómo es la competencia digital docente de los maestros de los Colegios Rurales Agrupados de la provincia de Albacete. En la fundamentación, se destaca que la incorporación de las TIC a la escuela rural se ve dificultada por la falta de habilidades docentes en TIC (Carnoy, 2004). Por ello es preciso conocer la frecuencia de uso que los docentes hacen de cada competencia porque ello determinará en gran medida si poseen las destrezas propias de cada una de ellas.

En el área de información y alfabetización informacional, los docentes hacen un uso constante de esta competencia, la cual implica habilidades para buscar, organizar y analizar información en función de su relevancia. Estas acciones se desarrollan de forma más habitual porque no requieren habilidades demasiado complejas y además los docentes necesitan hacer uso de ellas para tareas de la vida diaria, como por ejemplo acciones para almacenar información en pendrive para posteriormente recuperarla y hacer uso de ella. 
En el área de comunicación y colaboración, en términos generales, se percibe un uso limitado de esta competencia que implica interactuar y compartir recursos a través a través de comunidades digitales. Las causas pueden deberse a escasez de conocimientos y actitudes para interactuar digitalmente con fines profesionales, y a la falta de conciencia sobre el valor y el enriquecimiento profesional que implica el llevar acciones de colaboración e interacción digital.

En cuanto al área de seguridad, a modo general, los docentes llevan de manera regular acciones que implican protección personal, de datos y de identidad digital. Sin embargo, los docentes deben adquirir unas competencias más avanzadas que les capaciten para desarrollar acciones de protección en todos los entornos (salud, entorno, datos personales, dispositivos y contenidos digitales) con más exhaustividad.

Los ámbitos en los que se percibe mayor necesidad formativa son para la creación de contenidos digitales propios con derechos de autor, que implican competencias para crear contenidos en formatos diversos que integren recursos multimedia y además sean capaces de aplicar los derechos de autor. Es importante que los docentes adquieran estas habilidades porque ello va a posibilitar la adaptación del recurso y los contenidos a las características de los alumnos, además se facilita un aprendizaje constructivo y comprensivo adaptado a las necesidades, los intereses y el ritmo de aprendizaje de los alumnos.

Otro de los ámbitos en los que los docentes necesitan formarse es en la resolución de problemas técnicos en dispositivos tecnológicos y en el desarrollo de prácticas regulares de cuidado y protección de los dispositivos digitales que contribuyan a generar el menor impacto en el medio ambiente a través de medidas de ahorro energético. Si los docentes aprenden a integrar estas prácticas en su vida cotidiana y adquieren conciencia de su importancia, serán capaces de transmitir a los alumnos su valor.

Para finalizar, es preciso destacar el papel de la universidad, su misión es la de formar a los futuros maestros y es necesario que se les trasmita desde sus años académicos, la necesidad de formarse en competencias digitales. Ello implica que adquieran conocimientos, habilidades y actitudes referentes a las diferentes áreas competenciales. Igualmente, se les deben enseñar prácticas innovadoras sobre como integrar las tecnologías en el currículum. Un docente competente digitalmente, será capaz de dominar las habilidades de cada área competencial, y estará en condiciones de integrarlas en el currículum para que los alumnos las aprendan.

\subsection{Limitaciones}

La limitación por destacar en el desarrollo de este proyecto es la muestra de estudio, la cual no ha sido muy grande y por tanto no es representativa de la toda la población.

\subsection{Futuras líneas de investigación}

Como futuras líneas de investigación se pueden llevar a cabo proyectos más amplios y profundos, con una muestra más extensa, que traten de comparar la escuela rural y la urbana en cuanto a dotación tecnológica y nivel de competencia digital docente.

\section{ENLACES}

https://forms.gle/air9qVCZooTjPJHS7

http://www.educa.jccm.es/es/centros/tecnologia-educacion

https://www.juntadeandalucia.es/export/drupaljda/PlanEscuela.pdf

\section{REFERENCIAS BIBLIOGRÁFICAS}

Carnoy, M (2004, octubre). Las TIC en la enseñanza: posibilidades y retos. [Ensayo]. Universidad Abierta de Cataluña. https://bit.ly/2XmCL78

INTEF (2017). Marco Común de Competencia Digital Docente. Aprendelntef. https://bit.ly/3d0hRBd 
Elboj, C., Pulido, M.A. \& Welikala, T. (2013). Las tecnologías de la Información y la comunicación en la salida al aislamiento rural: el caso de Ariño. Revista electrónica de Geogrefía y Ciencias Sociales:Scripta Nova, 17(427). https://bit.ly/3eweZN1

Gutiérrez, I. (2011). Competencias del profesorado universitario en relación al uso de tecnologías de la información y comunicación: Análisis de la situación de España y propuesta de un modelo de formación [Tesis Doctoral. Universidad Rovira i Virgili]. TDX. https://bit.ly/3cT3jDo

Martín-Moreno, Q.(2002). Claves para la calidad de los centros educativos rurales. En M. Lorenzo et al. (Eds.), Liderazgo educativo y escuela rural, volumen 1 (pp.57-70). Grupo Editorial Universitario.

Salinas, A. y Sánchez, J. (2009). La superación de la brecha digital en las Escuelas Rurales de Chile. En J. Sánchez (Ed.), Nuevas Ideas en Informática Educativa (pp.157-164). Universidad de Chile. https://bit.ly/2TAqzi1

Warschauer, M. (2008). Whither the digital divide? In D.L. Kleinman, K.A.Cloud-Hansen, C. Matta \& J. Handesman (Eds.), Controversies in science \& technology: from climate to chromosomes (pp.140-150). Hardcover. https://bit.ly/2TydWDW

\section{INFORMACIÓN SOBRE LA AUTORA}

\section{María del Pilar Ruiz Ruiz \\ CRA de Castilla-La Mancha}

Licenciada en Pedagogía por la Universidad de Murcia (2006/2011). Máster en Formación del Profesorado: Especialidad Orientación Educativa (2016). Máster en Tecnología Educativa ELearning y Gestión del Conocimiento (2019). Actualmente ejerzo de Orientadora Educativa en un colegio y CRA de Castilla-La Mancha. CEIP San Antonio de Portaceli, en Sigüenza (Gudalajara) y CRA Sierra Ministra, Alcolea del Pinar (Gudalajara). El interés por el uso de las TICS en los colegios rurales agrupados me llevó a centrar mi línea de investigación en las tecnologías en el medio rural, destacando la disponibilidad de materiales y su uso, así como las competencias de los docentes de estos centros.

\section{@@(@)}

Los textos publicados en esta revista están sujetos a una licencia de Reconocimiento 4.0 España de Creative Commons. Puede copiarlos, distribuirlos, comunicarlos públicamente y hacer obras derivadas siempre que reconozca los créditos de las obras (autoría, nombre de la revista, institución editora) de la manera especificada por los autores o por la revista. La licencia completa se puede consultar en:Licencia Creative Commons Atribución-NoComercial-Compartir por igual 4.0 Internacional. 